\title{
Microbial Resistance Caused by Antibiotic Prophylaxis with Amoxicillin in a Male Infant with Vesicoureteral Reflux
}

\author{
Taniguchi K', Kii S², Oka M³ , Uemichi K ${ }^{4}$
}

\begin{abstract}
Vesicoureteral reflux is a common condition in infants with urinary tract infection. Although antibiotic prophylaxis to prevent recurrent urinary tract infection is widely performed, its effectiveness remains controversial. Herein we report a sevenmonth-old boy with vesicoureteral reflux. Antibiotic prophylaxis with amoxicillin caused microbial resistance accompanied by recurrent urinary tract infection. Subsequent antibiotic prophylaxis with sulfamethoxazole-trimethoprim was effective until spontaneous remission of vesicoureteral reflux occurred. Sulfamethoxazole-trimethoprim, but not amoxicillin, should be considered as an agent for antibiotic prophylaxis in infants with vesicoureteral reflux.
\end{abstract}

Key words: antibiotic prophylaxis; microbial resistance; vesicoureteral reflux

\section{INTRODUCTION}

U inary tract infections are common in infants, ${ }^{1}$ and can reflect underlying abnormalities like vesicoureteral reflux (VUR)., ${ }^{1,2}$ Although antibiotic prophylaxis to prevent recurrent urinary tract infection is widely performed, its effectiveness remains controversial. ${ }^{1,3-9}$ Herein we report a seven months old boy who was diagnosed with urinary tract infection and VUR. Antibiotic prophylaxis with amoxicillin caused microbial resistance accompanied by recurrent urinary tract infection. Subsequent antibiotic prophylaxis with sulfamethoxazole-trimethoprim (ST) was effective until spontaneous remission of VUR occurred.

\section{Case Report}

A seven months old infant presented with high fever. Urinary tract infection was diagnosed on the basis of both leukocyturia and a single uropathogenic organism in the urine. Microscopic examination revealed gram-positive bacteria. A culture examination revealed Enterococcus faecalis at $1 \times 105 / \mathrm{mL}$ in catheterised urine. He received intravenous administration of ampicillin $(50 \mathrm{mg} / \mathrm{kg}$, three times a day) for eight days, followed by oral administration of amoxicillin (10 $\mathrm{mg} / \mathrm{kg}$, three times a day) for six days. The treatment was effective for fever reduction. Radiological examinations were performed to investigate certain underlying conditions. Ultrasonography revealed hydronephrosis (Figure 1a). Voiding cysto-urethrography showed grade III bilateral VUR (Figure 2a). Other anomalies including
${ }^{1}$ Dr. Kazuto Taniguchi, MD, PhD; ${ }^{2}$ Dr. Sakumo Kii, MD; Both from Tara Town Hospital, Tara, Japan; ${ }^{3} \mathrm{Dr}$. Masafumi Oka, MD, PhD, Department of Paediatrics, Ureshino Medical Centre, Ureshino, Japan; ${ }^{4}$ Dr. Kazuyasu Uemichi, MD, Tara Town Hospital, Tara, Japan.

\author{
Address for correspondence \\ Kazuto Taniguchi, \\ Tara Town Hospital, 1520-12, Tara, \\ Saga 849-1602, \\ Japan \\ E-mail: tgene1@po.hagakure.ne.jp
}

\author{
Acknowledgements: None \\ Funding: Nil \\ Conflict of Interest: None \\ Permission from IRB: Not Submitted
}

\section{How to cite}

Taniguchi K, Kii S, Oka M, Uemichi K. Microbial Resistance Caused by Antibiotic Prophylaxis with Amoxicillin in a Male Infant with Vesicoureteral Reflux. J Nepal Paediatr Soc 2018;38(3):196-9.

doi: http://dx.doi.org/10.3126/jnps.v38i3.22662

Submitted on: 2019-02-11

Accepted on: 2020-02-12

This work is licensed under a Creative Commons Attribution 3.0 License.

\section{(c) (i)}


posterior urethral valve, ureteropelvic junction stricture, and renal atrophy were not detected. Subsequently, he received antibiotic prophylaxis with amoxicillin $(5 \mathrm{mg} / \mathrm{kg}$, once a day) to reduce the risk of recurrent urinary tract infection.

He suffered recurrence of urinary tract infection at one year of age. A culture examination revealed Klebsiella oxytosa at $1 \times 10^{5} / \mathrm{mL}$ in catheterised urine. He received intravenous administration of ceftriaxone $(60 \mathrm{mg} / \mathrm{kg}$, once a day) for one day, followed by oral administration of amoxicillin (10 mg/kg, three times a day) for seven days. The treatment was effective and he became asymptomatic. Subsequently, he received antibiotic prophylaxis with ST $(10 \mathrm{mg} / \mathrm{kg}$ sulfamethoxazole plus 2 $\mathrm{mg} / \mathrm{kg}$ trimethoprim, once a day).

Technetium 99m dimercaptosuccinic acid (99mTcDMSA) scintigraphy detected no abnormalities (data not shown). Voiding cystourethrography revealed grade II right VUR (Figure $2 \mathrm{~b}$ ) at 21 months of age. Antibiotic prophylaxis was stopped after improved VUR was confirmed. Ultrasonography demonstrated that hydronephrosis was improved at 22 months of age (Figure 1b) and had disappeared at 25 months of age (Figure 1c). No side effects of ST treatment were noted. He has not suffered from recurrence for $>30$ months since stopping antibiotic prophylaxis.
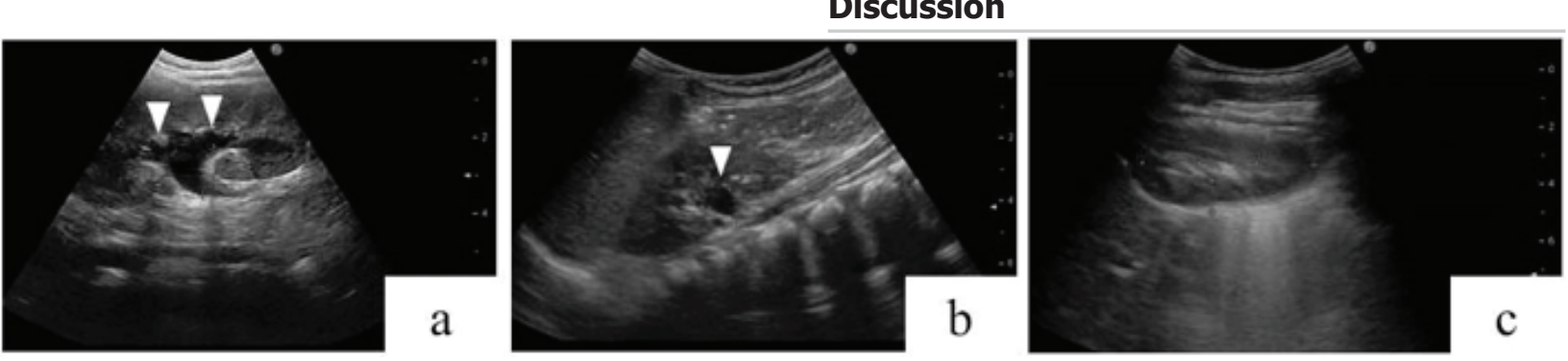

Fig 1: Ultrasonography of the kidney. (a) Ultrasonography revealed hydronephrosis (arrowheads) at 7 months of age. (b, c) The hydronephrosis was improved at 22 months of age (b) and had disappeared at 25 months of age (c)
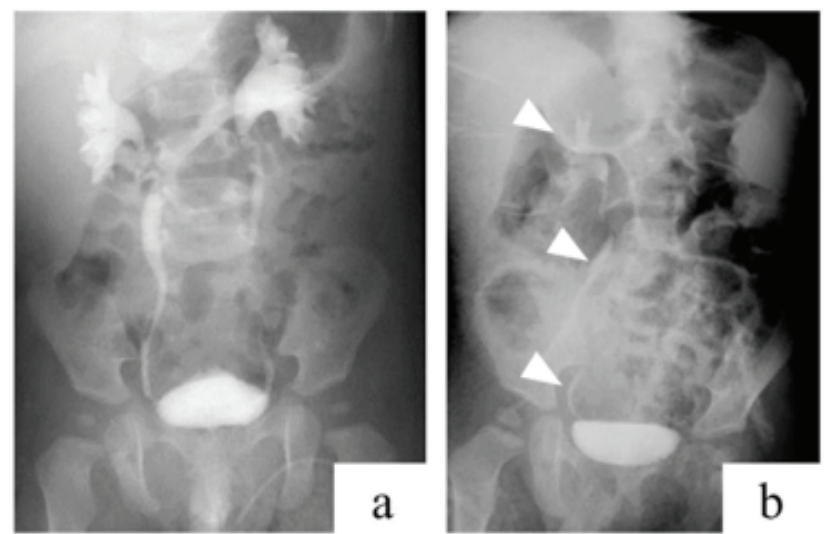

Fig 2: Voiding cystourethrography. (a) Voiding cystourethrography revealed VUR at seven months of age. (b) The VUR was improved (arrow heads) at 21 months of age

Table 1: Recent reports on antibiotic prophylaxis

\begin{tabular}{lcccc}
\hline Reference & Number of patients & Age & VUR grades & Stance \\
\hline Garin et al. $^{3}$ & 218 & $3 \mathrm{~m}-18 \mathrm{y}$ & I-III & Disapproval \\
\hline Conway et al. $^{4}$ & 611 & $<6 \mathrm{y}$ & I-V & Disapproval \\
\hline Pennesi et al. $^{5}$ & 100 & $<30 \mathrm{~m}$ & II-IV & Disapproval \\
\hline Montini et al. $^{6}$ & 338 & $2 \mathrm{~m}-7 \mathrm{y}$ & I-III & Disapproval \\
\hline Roussey-Kesler et al. $^{7}$ & 225 & $1 \mathrm{~m}-3 \mathrm{y}$ & I-III & Approval \\
\hline Craig et al. $^{8}$ & 576 & $<18 \mathrm{y}$ & I-III & Approval \\
\hline RIVUR Trial Investigators $^{9}$ & 607 & $2-71 \mathrm{~m}$ & I-IV & Approval \\
\hline
\end{tabular}

VUR: vesicoureteral reflux; m: months; y: years. 
We observed two important clinical issues in our patient: antibiotic prophylaxis with amoxicillin caused microbial resistance in an infant with VUR, and antibiotic prophylaxis with ST was effective in preventing recurrent urinary tract infection until spontaneous remission of VUR occurred.

Regarding the first issue, antibiotic prophylaxis with amoxicillin caused microbial resistance in our patient. Amoxicillin is often chosen as an agent for antibiotic prophylaxis to prevent recurrent urinary tract infection in infants with VUR in Japan. We chose amoxicillin because it allowed our patient to recover favorably from the urinary tract infection. Although amoxicillin covered $E$. faecalis in our patient, it did not cover $K$. oxytosa, another major microbial pathogen for urinary tract infections in infants. ${ }^{1}$ Although amoxicillin was adequate for treatment of the urinary tract infection in the acute phase, it was inadequate for antibiotic prophylaxis in our patient. Thus, an agent for antibiotic prophylaxis should cover $K$. oxytosa as well as $E$. faecalis.

For the second issue, ST was useful as an agent for antibiotic prophylaxis until spontaneous remission of VUR occurred. ST did not allow $E$. faecalis and $K$. oxytosa, or Escherichia coli as the principal microbial pathogen for urinary tract infection, to cause recurrence. Previous reports on antibiotic prophylaxis described oral administration of ST to prevent recurrent urinary tract infection in children. ${ }^{3-10}$ Selection of ST, rather than amoxicillin, after the initial urinary tract infection would have been preferable in our patient.

The American Academy of Paediatrics previously recommended antibiotic prophylaxis for VUR in infants and young children aged two months to two years, until completion of imaging studies like ultrasonography, voiding cystourethrography, and 99mTc-DMSA scintigraphy.10 Recently, however, there has been intense discussion on the effectiveness of continuous antibiotic prophylaxis in children with VUR. Some groups do not support antibiotic prophylaxis, ${ }^{3-6}$ as summarised in Table 1. Furthermore, Conway et al. ${ }^{4}$ indicated that antibiotic prophylaxis was associated with an increased risk of resistant infection. Meanwhile, other groups support antibiotic prophylaxis, ${ }^{7-9}$ as also summarised in Table 1. Roussey-Kesler et al. ${ }^{7}$ investigated 225 patients aged one month to three years with grade I-III VUR, and reported that antibiotic prophylaxis may be effective in boys with grade III VUR. Craig et al. ${ }^{8}$ investigated 576 patients aged $<18$ years with grade I-III VUR, as well as patients without VUR, and reported that antibiotic prophylaxis was associated with a $6 \%$ decrease in frequency of recurrence. The RIVUR Trial Investigators ${ }^{9}$ examined 607 patients aged two to 71 months of age with grade I-IV VUR, and reported that antibiotic prophylaxis substantially reduced the risk of recurrence by $50 \%$. Consequently, the optimal strategy remains unclear. Further accumulation of cases like the current patient is essential to clarify this point.

\section{Conclusions}

ST is useful for antibiotic prophylaxis to prevent recurrent urinary tract infection in infants with VUR, while amoxicillin is associated with a risk of microbial resistance. ST should be initially considered as an agent for antibiotic prophylaxis in infants until spontaneous remission of VUR occurs.

\section{References}

1. Arshad $M$, Seed PC. Urinary tract infections in the infant. Clin Perinatol. 2015;42:17-28. DOI: 10.1016/j. clp.2014.10.003.

2. Arena $S$, lacona R, Impellizzeri P, Russo T, Marseglia L, Gitto E, et al. Physiopathology of vesico-ureteral reflux. Ital J Pediatr. 2016;42:103. DOI: 10.1086/ s13052-016-0316-x.

3. Garin EH, Olavarria F, Garcia Nieto V, Valenciano B, Campos A, Young L. Clinical significance of primary vesicoureteral reflux and urinary antibiotic prophylaxis after acute pyelonephritis: a multicenter, randomized, controlled study. Pediatrics. 2006;117:626-32. DOI: 10.1542/peds.2005-1362.

4. Conway PH, Cnaan A, Zaoutis T, Henry BV, Grundmeier RW, Keren R. Recurrent urinary tract infections in children: risk factors and association with

prophylactic antimicrobials. JAMA. 2007;298:179-86. DOI: 10.1001/jama.298.2.179.

5. Pennesi M, Travan L, Peratoner L, Bordugo A, Cattaneo $A$, Ronfani $L$, et al. Is antibiotic prophylaxis in children with vesicoureteral reflux effective in preventing pyelonephritis and renal scars? A randomised controlled trial. Paediatrics. 2008;121:1489-94. DOI: 10.1542/peds.2007-2652.

6. Montini G, Rigon L, Zucchetta P, Fregonese F, Toffolo A, Gobber D, et al. Prophylaxis after first febrile urinary tract infection in children? A multicenter, randomized, controlled, noninferiority trial. Pediatrics. 2008;122:1064-71. DOI: 10.1542/peds.2007-3770.

7. Roussey-Kesler G, Gadjos V, Idres N, Horen B, Ichay $\mathrm{L}$, Leclair MD, et al. Antibiotic prophylaxis for the prevention of recurrent urinary tract infection in children with low grade vesicoureteral reflux: results from a prospective randomised study. J Urol. 2008;179:6749. DOI: 10.1016/j.juro.2007.09.090. 
8. Craig JC, Simpson JM, Williams GJ, Lowe A, Reynolds GJ, McTaggart SJ, et al. Antibiotic prophylaxis and recurrent urinary tract infection in children. $\mathrm{N}$ Engl J Med. 2009;361:1748-59. DOI: 10.1056/ NEJMoa0902295.

9. Hoberman A, Greenfield SP, Mattoo TK, Keren R, Mathews R, Pohl HG et al. Antimicrobial prophylaxis for children with vesicoureteral reflux. N Engl J Med. 2014;370:2367-6. DOI: 10.1056/NEJMoa1401811.

10. American Academy of Paediatrics, Committee on Quality Improvement, Subcommittee on Urinary Tract Infection.Practice parameter: the diagnosis, treatment, and evaluation of the initial urinary tract infection in febrile infants and young children. Paediatrics. 1999;103(4Pt1):843-52. DOI: 10.1542/ peds.103.4.843. 